\title{
3. Blockchain and Darwin
}

\section{$1 \quad$ FIRST, DARWIN}

In spite of the fundamental character of his literature, the transposition of Darwin's work to modern economies does not explain how new technologies appear. The answer to this enigma can be found in the work of W. Brian Arthur, who explained that new technologies result from unexpected combinations. ${ }^{1}$ Blockchain, a combination of the technologies I mentioned in the first chapter, is very much a combinatorial technology. That being said, Darwin's findings do explain how living organisms (e.g., technologies) evolve and survive. They are thus crucial for understanding how blockchain - now that it exists - could develop.

Darwin started The Origin of Species by explaining that survival is more ferocious between different varieties of the same species than between different species. ${ }^{2}$ Indeed, varieties consume the same food and are exposed to the same dangers, so the struggle for survival directly depends on the actions of others. As a result, only the species that are capable of adapting survive. Of course, one sees "nothing of these slow changes in progress." Mutations happen over time, creating what Darwin calls natural selection. But natural selection is prevented when species grow in an environment protected by barriers. One may indeed observe fewer variations because the need to "diverge in structure, habits, and constitution"4 to survive among other species tends to be significantly reduced. Since "we are much too ignorant in regard to the whole economy of any one organic being to say what slight modifications would be of importance or not," ${ }^{\prime 5}$ these barriers end up putting the species at risk.

1 See W. Brian Arthur, The Nature of Technology: What It Is and How It Evolves (Free Press, 2009).

2 Charles Darwin, On the Origin of Species (John Murray of Albemarle Street, 1859): 52.

3 Id. at 84 .

4 Id. at 128 .

5 Id. at 195. 
Variations cause organisms to change themselves to "economize in every part." ${ }^{10}$ As a result, a characteristic that is useful in one specific environment can become detrimental when the organism is moved to another. ${ }^{7}$ On the contrary, the lack of a certain characteristic in a new environment may also cause the species to disappear. In the end, "natural selection acts solely by and for the good of each." " It is a strictly utilitarian process, without morality. ${ }^{9}$ The strongest species and varieties live, and the weakest die. ${ }^{10}$

So, what is the lesson for blockchain? One must distinguish between two periods. The first period is one of natural selection between blockchain varieties. Before they compete with centralized applications, blockchain varieties undergo different mutations that could prove useful for that later stage of competition. These mutations occur at a time when blockchain is not protected by immutable barriers, whether natural or regulatory. They cannot be engineered; "no man can predict"11 which characteristics will lead specific varieties to prevail.

The second stage is that of maturity. The most efficient blockchain varieties will logically thrive and, as a result, they will gradually enter the territory of centralized applications. The blockchain environment will then change. Competition between these two species - decentralized and centralized ones will be engaged, and certain characteristics of blockchain will prove central to its survival. If several of these characteristics have been eliminated artificially before blockchain's varieties have reached maturity, their chance of survival will be significantly affected. For example, removing blockchain's immutability could cause the elimination of a key differentiating feature, compared to centralized applications where a few individuals control data. This is the danger of evolutionary systems involving human beings capable of changing trajectories voluntarily, which animals cannot do. ${ }^{12}$ Manufacturing evolution is a perilous game.

\author{
Id. at 147 . \\ Id. at 172 . \\ Id. at 201. \\ Id. at 199 . \\ Id. at 244 . Also, stressing that "natural selection acts by life and death." Id. at \\ Id. at 126. \\ 2 George Soros, "Fallibility, Reflexivity, and the Human Uncertainty Principle,"
} 194. Journal of Economic Methodology 20, no. 4 (2013): 319 (explaining that the main distinction "between natural and social science consists of the presence or absence of thinking participants who have a will of their own"). 
Blockchain's unique characteristics give rise to numerous use cases. Before exploring them, it is important to note that only platform blockchains can be used for different purposes, while monocentric blockchains are tied to one specific use.

\subsection{Monocentric and Platform Blockchains}

Not all blockchains allow software to run on top of the constitutional layer. Monocentric blockchains permit only one application. The Bitcoin blockchain is a good example, even if several initiatives have been announced to make it more versatile..$^{13}$ To this day, the Bitcoin blockchain is almost exclusively used for the Bitcoin cryptocurrency. This is due to the fact that its programming language cannot easily be exploited for other uses. Some are also questioning the willingness of Bitcoin miners to devote computing power to other types of transactions. This type of blockchain gets its entire value from the application for which it was created. ${ }^{14}$

Platform blockchains allow an unlimited number of applications to be added on top of layer 1. Ethereum is a great example of a platform blockchain, as anyone can upload a program onto the constitutional layer and leave it to self-execute. This freedom primarily exists because it uses Solidity as a programming language, ${ }^{15}$ "allowing anyone to write smart contracts, and decentralized applications where they can create their own arbitrary rules for

13 "Bitcoin to Get Smart Contracts," Trustnodes, August 7, 2019, https://perma .cc/85HR-9BWF; Alyssa Hertig, "Pieter Wuille Unveils 'Miniscript,' A New Smart Contract Language for Bitcoin," CoinDesk, August 20, 2019, https://perma.cc/97D4 -BYH9.

14 See Paul Vigna and Michael J. Casey, The Truth Machine: The Blockchain and The Future of Everything (St. Martin's Press, 2018): 99 ("How do tokens work? Just as Bitcoin's protocol steers users and participants into certain actions that serve the community's interest - in its case, creating a secure, reliable ledger that all can trust - the programs that run tokens incorporate incentives and constraints that encourage certain pro-social behavior. A new concept-token economics-is emerging. It encapsulates the idea that we can embed into these 'programmable' forms of money a way to steer communities toward desired common outcomes. Tokens might help us solve the Tragedy of the Commons. In other words, they could be a big deal.")

15 Carla L. Reyes, "If Rockefeller Were a Coder," George Washington Law Review 87, no. 373 (2019): 386 ("The Ethereum protocol supports its own coding language, Solidity, intended to enable software developers to write complex smart contracts more simply. Furthermore, Ethereum contracts are stateful and Turing-complete- they have 'memory that they will remember the next time they are called and ... they can have loops."”) 
ownership, transaction formats, and state transition functions." 16 Here, the blockchain code can be described as a "fat protocol," "since the success of the application layer drives further speculation at the protocol layer." ${ }^{17}$ Inversely, should a blockchain layer 1 encounter difficulties (or concentration problems), this would create a disincentive to use it.

As far as antitrust enforcement is concerned, it is easy to see how platform blockchains are prone to more infringements, as power at the fat protocol level can be more or less easily leveraged against other layers (depending on blockchain characteristics). I will return to this later in the book. One should not conclude, however, that monocentric blockchains are immune from antitrust issues. As we will see, market definition might be easier, leading to quicker antitrust enforcement activities.

\subsection{Blockchain 1.0, 2.0 and 3.0}

One can use blockchain for several distinct purposes, so let me propose a rudimentary nomenclature. For that purpose, I adopt a slightly modified version of the one offered by Melanie Swan in 2015, according to which one can distinguish between three categories of blockchain applications. The first (blockchain 1.0) is cryptocurrency, where blockchain tokens are used as coins. ${ }^{18}$ The second (blockchain 2.0) is smart contracts. Here, blockchains are executing automated transactions between users. ${ }^{19}$ The third (blockchain 3.0) encompasses all other blockchain uses, including peer-to-peer ridesharing, social media, online research and more. ${ }^{20}$ In fact, blockchain 3.0 includes alternatives to the products and services that are generally described as the "sharing economy."

16 Ethereum, "A Next-Generation Smart Contract and Decentralized Application Platform," GitHub, https://perma.cc/G72K-YUVJ.

17 Joel Monegro, "Fat Protocols," Union Square Ventures, August 8, 2016, https:// perma.cc/KGS6-7HLN.

18 Melanie Swan, Blockchain: Blueprint for A New Economy (O'Reilly Media, 2015): 1-8.

19 "A smart contract is a set of promises, specified in digital form, including protocols within which the parties perform on these promises." Nick Szabo, "Smart Contracts," University of Amsterdam, https://perma.cc/5NF3-R6N3. Ethereum, "A Next-Generation Smart Contract and Decentralized Application Platform," GitHub, https://perma.cc/G72K-YUVJ (describing smart contracts as "complex applications involving having digital assets being directly controlled by a piece of code implementing arbitrary rules.") For an overview of how smart contracts work, see Kevin Werbach and Nicolas Cornell, "Contracts Ex Machina,” Duke Law Journal 67, no. 2 (2017): 319.

20 See Melanie Swan, Blockchain: Blueprint for A New Economy (O'Reilly Media, 2015): 29-70. 
When it comes to antitrust, each type of blockchain comes with unique challenges. Blockchain 1.0 competes with states' currencies; it may lead to the disruption of their prerogatives. This alone will likely lead to heavy scrutiny from antitrust agencies, such as the antitrust investigation introduced by the European Commission against Diem - initially called Libra - Facebook's cryptocurrency. ${ }^{21}$ Blockchain 2.0 applications, as we shall see in Chapter 9, may be used for setting up antitrust practices, as smart contracts create trust between participants - which is central to their willingness to engage in transactions (including illegal ones). Lastly, blockchain 3.0 comes with some challenges similar to those encountered with centralized platforms.

Here goes the general overview that I will explore further on. If one thing, Darwin teaches us that competition is utilitarian, meaning that only the end result matters for the varieties and species submitted to it. At times, it may imply anti-competitive practices. But for now, I want to focus on the competition between all these different blockchain types and analyze the procompetitive dynamism created between them.

\section{A DARWINIAN ENVIRONMENT}

The current blockchain ecosystem is highly dynamic. Varieties compete with each other through a combination of ideological orientations and business objectives that translate into technical characteristics. ${ }^{22}$ Several trends are emerging from that competition. Projects are being built on top of others, creating a true Darwinian environment. Let us take a closer look.

\subsection{A Dynamic Ecosystem}

One can measure the dynamism of the blockchain ecosystem in many ways. Generally, two forces may encourage the adoption of a new technology: a top-down "institutional" force and a bottom-up "civilian" force. Regarding the first, consulting firm PwC found in 2018 that 84 percent of 600 surveyed executives from 15 territories said they were involved in blockchain experi-

21 Thibault Schrepel, "Libra: A Concentrate of Blockchain Antitrust," Michigan Law Review Online 118, no. 160 (2020).

22 Jeffery Atik and George Gerro, "Hard Forks on the Bitcoin Blockchain: Reversible Exit, Continuing Voice," Stanford Journal of Blockchain Law \& Policy 1 (2018): 35 ("Exit, for miners at least, is reversible at low cost. The post-fork history seems to demonstrate that some miners simply mined the more profitable blockchain. Other miners remained on the same network for political reasons.") 
mentation. ${ }^{23}$ And while it is nearly impossible to list all the institutions that are already using it, a few big names spring to mind. They include the likes of Facebook, Wal-Mart, Amazon, PayPal, Bank of America, Microsoft, Google, HTC, IBM, ING, Intel, JP Morgan, the U.S. Commodity Futures Trading Commission, the Chicago Mercantile Exchange, Stanford University, Harvard University, Visa, Samsung, Tesla, Mastercard, Comcast and Fidelity. ${ }^{24}$ For example, Wal-Mart is working with IBM on using blockchain to increase supply chain transparency by tracing food..$^{25}$ Microsoft has been developing blockchain products to allow its customers to reduce fraud and verify transactions. ${ }^{26}$ Samsung is now offering access to its Samsung Blockchain Wallet to connect customers "to a world of decentralized apps." ${ }^{.27}$ Amazon offers more than 70 blockchain services to its customers ${ }^{28}$ and uses blockchain to allow all supply chain participants to access real-time supply chain data. ${ }^{29}$ PayPal is allowing cryptocurrency trading for all its users. ${ }^{30}$ Pfizer is tracking drugs from manufacturing to pharmacies using MediLedger. ${ }^{31}$ Comcast uses it to offer a new targeted ads platform. ${ }^{32}$ Boeing is selling airline parts on Honeywell,

${ }^{23}$ PwC, "Blockchain Is Here. What's Your Next Move?," https://perma.cc/TSJ9 -UKHQ.

24 David Allessie, Maciej Sobolewski and Lorenzino Vaccar, "Blockchain for digital government," Publication Office of the European Union (2019): 14. For more, see, for instance, Michael del Castillo, "Blockchain 50: Billion Dollar Babies," Forbes, https://perma.cc/P7SU-DYUU; and Andrew Singer, "2019's Top 10 Institutional Actors in Crypto," Cointelegraph, February 1, 2020, https://perma.cc/QU4U-HZ73.

25 The Leadership Network, "How Walmart used blockchain to increase supply chain transparency," The Leadership Network, January 22, 2020, https://perma.cc/ NH97-YAYS.

${ }^{26}$ Microsoft Azure, "Blockchain Technology and Applications," https://perma.cc/ D8B5-YQXG.

27 Samsung, "Samsung Blockchain, Simple and Trust Proven," https://perma.cc/ 33G6-GGHG.

28 Amazon Web Services, "Blockchain on AWS: Enterprise Blockchain Made Real," https://perma.cc/3NKA-PLQM.

29 Dan Berthiaume, "Report: New Amazon Blockchain Solution Targets Product Authenticity," Chain Store Age, April 6, 2020, https://perma.cc/M55Q-FEKL.

30 Robert Stevens, "PayPal Launches Cryptocurrency Trading for All US Users," Decrypt, November 12, 2020, https://perma.cc/84CE-XZU4.

${ }_{31}$ Gertrude Chavez-Dreyfuss, "Companies in Pharmaceutical Supply Chain Develop System to Track Counterfeit Drugs," Reuters, February 21, 2020, https:// perma.cc/E4RY-FTMQ.

32 Jeff Baumgartner, "How Comcast Will Use Blockchain Tech for Targeted Ads," Light Reading, January 24, 2019, https://perma.cc/7NHU-ASAK. 
a blockchain-based marketplace, for over $\$ 1$ billion. ${ }^{33}$ Hardly a single week passes without major announcements in the field.

Then there are also Canada, Mexico, the United States, Argentina, Austria, Denmark, Estonia, Georgia, Ireland, Italy, Latvia, Lithuania, Luxembourg, Malta, the Netherlands, Slovenia, Spain, Sweden, Switzerland, Ukraine, the United Kingdom, Ethiopia, Kenya, Liberia, Mauritius, Nigeria, Rwanda, South Africa, Sierra Leone, Tanzania, Uganda, Zambia, China, Hong Kong, India, Malaysia, Singapore, South Korea, Thailand, the United Arab Emirates and Australia. ${ }^{34}$ These governments are using blockchain to facilitate the transparent administration of government contracts and public procurement procedures; transmit secure messages; implement and secure digital identity management; store health data; manage the land registry system; verify academic credentials; and soon, run their own cryptocurrencies. ${ }^{35}$

Regarding the "civilian" force, more than 1 billion transactions were recorded on public blockchains in 2019 alone, for a total exceeding 3 billion transactions in ten years. ${ }^{36}$ That number sounds impressive, but let me note that Visa alone records 150 million transactions every day. ${ }^{37}$ As for developers, over 7000 were active in 2019. But again, this must be put into perspective against the 6 million Android developers. ${ }^{38}$ The same goes for the existence of over 7000 blockchain companies listed by Crunchbase. ${ }^{39}$ There is thus much room for growth.

One can also measure blockchain dynamism from the inside, as opposed to merely analyzing adoption trends. Proof of Work continues to store the most value as far as protocols are concerned. The distribution of market capitalization by consensus algorithm showed an overwhelming 86.7 percent for Proof of Work in $2019 .{ }^{40}$ The Ethereum Foundation has, however, started its migra-

33 Michael del Castillo, "Honeywell Is Now Tracking \$1 Billion In Boeing Parts on A Blockchain," Forbes, March 7, 2020, https://perma.cc/HBG2-KHCC.

34 Lesa Moné, "Which Governments Are Using Blockchain Right Now?" ConsenSys, November 18, 2019, https://perma.cc/9H2M-UF3V.

35 See Kevin Helms, "China's Digital Currency Has Been Used in 3 Million Transactions Worth Over a Billion Yuan So Far," Bitcoin, January 1, 2021, https:// perma.cc/CK4G-BQV8; EU Blockchain Forum, "New Thematic Report: Central Bank Digital Currencies and a Euro for the Future," June 5, 2021, https://perma.cc/8UC6 -TWZC; "Digital Dollar," Coindesk, https://perma.cc/TJN6-5HL8.

${ }_{36}$ Pogorzelski, "Plus de 3 Milliards de Transactions Bitcoin et Crypto Réalisées en 10 Ans" Cryptonaute, February 25, 2020, https://perma.cc/7LRE-MRVS.

37 State of Adoption, "Report 2019/2020" (2020): 9, https://perma.cc/PZA9-X92Y.

38 Id. at 8.

39 Crunchbase, Blockchain Companies, May 26, 2021, https://perma.cc/KBF5 -KKWX.

40 State of Adoption, "Report 2019/2020" (2020): 28, https://perma.cc/PZA9 $-\mathrm{X} 92 \mathrm{Y}$. 
tion to Proof of Stake. Phase 0 of Ethereum 2.0 was launched in December 2020, while phases 1 and 2 will continue until 2022. ${ }^{41}$

Lastly, the distribution of investments is also a good indicator of where the blockchain ecosystem is headed. In 2019, finance protocols and applications (e.g., decentralized finance (DeFi), exchanges, fintech) generated the most venture returns. ${ }^{42}$ In early 2020, DeFi contracts passed $\$ 12$ billion (i.e., referring to the total value locked in DeFi), while several DeFi tokens have appreciated by 4000 percent this year alone. This has led to the creation of many blockchain remittance companies. ${ }^{43}$ Last, the interest in decentralized autonomous organizations (DAOs) has also grown significantly since an incident in 2016 when hackers stole $\$ 50$ million by exploiting software vulnerability. Over 1000 DAOs were created in 2019 alone. ${ }^{44}$ I will come back to those in due course.

\subsection{Today's Players}

At the time of writing this chapter, at the end of 2020, two blockchains appear to stand out over 4000: ${ }^{45}$ Bitcoin and Ethereum. As far as Bitcoin is concerned, the numbers are astronomical. Since publication of Satoshi Nakamoto's paper in October 2008 and the first transaction on January 12, 2009, Bitcoin passed the milestone of 500 million transactions in February 2020. ${ }^{46}$ Just over 450000 unique addresses were used on that blockchain during that same month. ${ }^{47}$ In 2019, the total number of transactions per day was between 300000 and $400000 .^{48}$

${ }^{41}$ See Rene Millman, "What is Ethereum 2.0 and Why Does It Matter?" Decrypt, December 1, 2020, https://perma.cc/MEV8-WP57 (explaining these three different phases).

42 State of Adoption, "Report 2019/2020," (2020): 7, https://perma.cc/PZA9 -X92Y.

43 See Blockdata, "Remittance Market \& Blockchain Technology," (2019), https:// perma.cc/4VVZ-K8UX.

44 State of Adoption, "Report 2019/2020," (2020): 143, https://perma.cc/PZA9 -X92Y.

45 See CoinMarketCap, “All Cryptocurrencies,” CoinMarketCap, https://perma.cc/ PGE2-S46S (listing 4166 active coins and tokens as of January 5, 2021).

46 "Total Number of Transactions," Blockchain.com, https://perma.cc/ZB5X -CPHL.

47 "Unique Addresses Used," Blockchain.com, https://perma.cc/DJQ7-VWKZ.

48 "Confirmed Transactions Per Day," Blockchain.com, https://perma.cc/45X6 -Z6VF. 
Ethereum has also grown significantly. As of April $2020,{ }^{49}$ over 700 million transactions had been recorded on the Ethereum chain since its launch in August $2015 .{ }^{50}$ In 2020, it also surpassed the $\$ 1$ trillion mark in settlements. ${ }^{51}$ Each day of 2019, the number of transactions executed fluctuated between 400000 and 1 million. ${ }^{52}$ The Ethereum blockchain had 88 million unique addresses in February 2020. That same month, over 800000 smart contracts were created, and 41 million automated transactions were concluded. ${ }^{53}$ Token adoption and awareness are also on the rise. ${ }^{54}$

Many other projects have attracted significant investments. EOS, a blockchain development platform, raised $\$ 4.1$ billion in $2018 .{ }^{55}$ Figure, a fintech player leveraging blockchain for debt financing, raised almost $\$ 1.2$ billion at the end of 2019. ${ }^{56}$ Blockchain and semiconductor company Bitmain had raised more than $\$ 1.5$ billion by the end of $2018 . .^{57}$ The Telegram Open Network, a peer-to-peer distributed storage and hosting system, raised $\$ 850$ million through an initial coin offering (ICO) ${ }^{58}$ while stock brokerage application Robinhood had raised more than $\$ 850$ million by the end of $2019 .{ }^{59}$ Wait, there's more. Digital currency exchange Coinbase raised over \$500 million by the end of 2018; ${ }^{60}$ while Bakkt, a regulated Bitcoin futures and custody exchange, was approaching $\$ 500$ million at the beginning of $2020 .{ }^{61}$ Also,

\footnotetext{
49 "Ethereum Total Transaction Count Chart," Blockchair.com, https://perma.cc/ SDL2-XZG2.

50 "Ethereum Transaction Hash (Txhash) Details, Ethereum (ETH) Blockchain Explorer,” Etherscan, https://perma.cc/63H6-A8PT.

${ }_{51}$ Michael McSweeney, "Charting Ethereum's Five-Year Journey by the Numbers," The Block, July 30, 2020, https://perma.cc/P2PE-KRKT.

52 "Total Number of Transactions Per Day," Etherchain.org, https://perma.cc/ 9K4A-Y2UC.

53 "Ethereum by the Numbers-February 2020," ConsenSys, March 2, 2020, https://perma.cc/UY9E-ZDCL.

54 According to the Financial Conduct Authority (FCA), 3.86 percent of the British general population owned cryptocurrencies in 2020, see Rebecca English et al., "Research Note, Cryptoasset Consumer Research 2020," Financial Conduct Authority, June 30, 2020: 5, https://perma.cc/XSS8-5EUW. The FCA also noticed an "increase in the percentage of those being aware of cryptocurrencies from $42 \%$ to $73 \%$ of adults," $i d$.

55 "EOS," Blockdata, https://perma.cc/72JB-9A83.

56 "Figure," Blockdata, https://perma.cc/4TLQ-D3DR.

57 "Bitmain," Blockdata, https://perma.cc/65FP-CE7W.

58 “Telegram Open Network," Blockdata, https://perma.cc/9LSH-86U6.

59 "Robinhood (Crypto)," Blockdata, https://perma.cc/T3GC-8HUF.

"Coinbase," Blockdata, https://perma.cc/D7CW-HFZF.

61 "Bakkt," Blockdata, https://perma.cc/3F2X-VG92. According to the FCA, 63 percent of tokens owner were using Coinbase in 2020, 15 percent Binance and 10 percent Kraken, see Rebecca English et al., "Research Note, Cryptoasset con-
} 
Noku, an all-in-one custom blockchain platform, raised over 300 million in 2018. ${ }^{62}$ Others - such as Kraken, ${ }^{63}$ a bitcoin and cryptocurrency trading platform; BitFury, ${ }^{64}$ a hardware and blockchain development solution; and Tron, a blockchain development platform for content sharing ${ }^{65}$ - each raised over $\$ 100$ million.

\subsection{What's Next? The Crystal Ball}

As the saying goes, "Prediction is hard, especially when it's about the future." Drawing a portrait of what future blockchain ecosystems will look like is a perilous exercise, but I will try nonetheless: blockchain will evolve to the point of competing with non-blockchain products and services. Centralized firms will slow it down, capturing part of its value by recentralizing it artificially. But the value created by decentralized blockchains at layer 1 will nonetheless end up transforming part of the world's economic transactions.

Numerous institutions are also making predictions. The World Economic Forum predicts that 10 percent of global gross domestic product (GDP) will be stored on blockchain by $2027 .{ }^{67}$ Bain \& Company has predicted in a study that by 2026, distributed ledger technology and blockchain will increase the volume of global trade by $\$ 1.1$ trillion from $\$ 16$ trillion today - a 6.9 percent increase. ${ }^{68}$ PwC's estimates are slightly higher: it attributes a $\$ 1.76$ trillion impact to blockchain, representing 1.4 percent of the world's total GDP. ${ }^{69}$ Lastly, Gartner prophesizes that blockchain will create $\$ 3.1$ trillion worth of business value by $2030 .^{70}$

sumer research 2020," Financial Conduct Authority, June 30, 2020, www.fca.org.uk/ publication/research/research-note-cryptoasset-consumer-research-2020.pdf, 19.

62 "Noku," Blockdata, https://perma.cc/T3E8-UE96.

63 "Kraken," Blockdata, https://perma.cc/CPV5-FNB6.

64 "BitFury," Blockdata, https://perma.cc/H4YQ-A9ZU.

65 "TRON," Blockdata, https://perma.cc/CP2F-KW5V.

${ }_{66}$ A sentence variously attributed to physicist Niels Bohr and baseball player Yogi Berra.

67 World Economic Forum, "Deep Shift: Technology Tipping Points and Societal Impact" (2015): 24.

68 "Blockchain Could Increase Global Trade Volumes by $\$ 1.1$ trillion by 2026 , Off the Current Base of \$16 trillion," Bain, October 22, 2018, https://perma.cc/Z8DX $-\mathrm{C} 3 \mathrm{KY}$.

69 Lujan Odera, "Blockchain Technology Is Set to Grow the Global GDP by $\$ 1.76$ Trillion In the Next Decade: PwC Report,” Bitcoin Exchange Guide, October 13, 2020 , https://perma.cc/NP4B-LPKN.

70 "Forecast: Blockchain Business Value, Worldwide, 2017-2030," Gartner, March 2, 2017, https://perma.cc/ATK2-3YHW. 
Prediction growth often amounts to reading tea leaves. What matters here is not the exact numbers, but the idea that blockchain could enable new transactions on top of transforming existing ones. One must seek to understand why that is. Gartner provides us with what I find a convincing explanation of blockchain adoption.

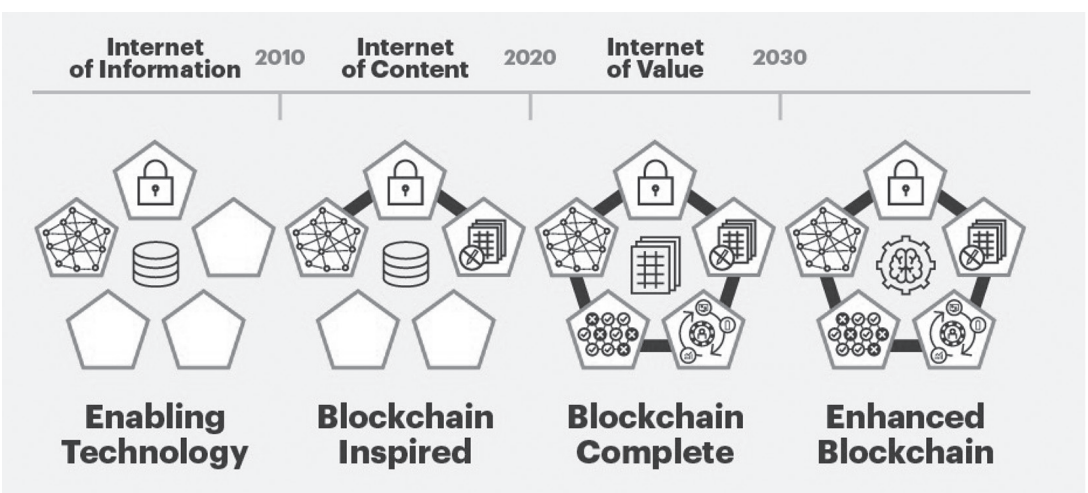

Source: Based on Kasey Panetta, "The 4 Phases of the Gartner Blockchain Spectrum," Gartner, October 14, 2019, https://perma.cc/HY96-9UKF.

Figure $3.1 \quad$ Stages of blockchain adoption - Gartner: 2019

According to the company, the 2000s were the "information age," allowing market players to disseminate their offline content on the Internet. Technological advances such as improved Internet speed led to the "content society." But blockchain is different; it is a transactional technology. Should it ever impact society at large, it will transform the way we interact with each other (eliminating various intermediaries).

How exactly blockchain will achieve that remains to be seen. In fact, the technology is undergoing an evolutionary process that is accelerated by its capacity to fork. There will be (most likely) no eureka moments (i.e., "killer app") in its development, but rather a sequence of incremental changes in a more or less consistent and chaotic direction. ${ }^{71}$

In the end, these technical evolutions will influence the financial support blockchain will receive. For that reason, I am not quite sure (to say the least)

71 Discussing chaotic evolutions, see James Gleick, Chaos: Making a New Science (Vintage Books, 1996). 
that one can prophesy exactly when blockchain will move from one phase to the other, but Gartner's logical steps seem to make sense.

The company describes the years 2020-2022 as the "trough of disillusionment," following the craze of the 2010s (Phase 1). ${ }^{72}$ It further predicts that the decade we are just entering will be one of "large focus investments" (Phase 2); followed by global and large-scale value-add solutions between 2027-2030 (Phase 3). ${ }^{73}$ And eventually, the 2030 s could be the years of blockbuster ecosystems coupled with other technologies such as artificial intelligence and the Internet of Things (I will explain how blockchain could help these technologies in Chapter 15). That is, if blockchain and law become allies.

\section{CHAPTER SUMMARY AND BEYOND}

In this chapter, I have first discussed Darwin's findings. In The Origin of Species, he observed that competition occurs between varieties of the same species before moving on to competition between different species. Transposed to digital technologies and ecosystems, this means that competition first happens between blockchain's applications before moving between blockchain and non-blockchain applications.

With that in mind, I then explored blockchain ecosystems and verify the Darwinian hypothesis in the field. I showed that blockchain can be monocentric or platforms, depending on whether apps and services can function on top of them. I then distinguished between three different types of uses: blockchain 1.0 for cryptocurrencies, blockchain 2.0 for smart contracts and blockchain 3.0 for all other decentralized applications.

The next part of the chapter discussed how fast blockchain could challenge incumbent platforms. I started by exploring blockchain's increasingly fast adoption by both large institutional players and civilians. I discussed how the technology is being used and how it compares to non-blockchain services. Guessing what could happen in the coming years - always a dangerous exercise - I went back to Darwin's theory of evolution, explaining that looking for blockchain's killer app is probably a mistake; and, in any case, that anticipating the day (or week, or month, or year) when it will appear is even more doubtful.

72 See "Blockchain Technology Spectrum: A Gartner Theme Insight Report," Gartner (2018): 10. Following a survey of 1386 senior executives in a dozen countries, more than 50 percent of respondents said that "blockchain technology has become a critical priority for their organizations in 2019-a 10-point increase over last year," “Deloitte's 2019 Global Blockchain Survey," Deloitte (2019): 3.

73 "Blockchain-Based Transformation: A Gartner Trend Insight Report," Gartner: 7. 
In the following chapter, I address the elephant in the room: what are most blockchains competing against, and what characteristics might enable them to survive against non-blockchain applications? In short, I discuss the reason(s) behind the blockchain craze and show that blockchains compete by translating ideology into technical advancements. 